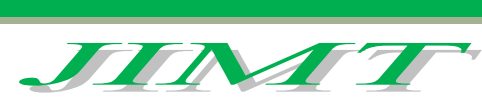

Jurnal Ilmiah Matematika

ISSN : $2450-766 X$

\title{
MISCONCEPTIONS IN SOLVING INDEFINITE INTEGRALS FOR NONELEMENTARY FUNCTIONS USING THE TAYLOR SERIES
}

\author{
Mohammad Lutfi1 \\ 1STT MIGAS Balikpapan \\ 1Politeknik Ilmu Pelayaran Balikpapan \\ Email: lutfi_plhld@yahoo.co.id
}

\begin{abstract}
The research aims to obtain informations related to the misconceptions in solving indefinite integrals for nonelementary functions using the Taylor series. The critical analysis with focus on the integral solution is used to reveal the misconceptions. The research was conducted by analysing integrand and/or the part of integrand such that the Taylor series could be applied and meet the requirements to integrable, and then integrating the Taylor series. The results revealed that pay no attention to the remainder term so that integration of the Taylor series of $f$ function is not represent of $f$ function itself, selection of the Taylor series about $x=0$ or $x \neq 0$ is not suitable to the integrand form, the way to obtain the Taylor series by supposition technique, and assumption that the Taylor series expansion of $f(x)$ equal to the Taylor series expansion of $g(x)$ divided by the Taylor series expansion of $h(x)$ could be applied for all condition are the misconceptions in this research.
\end{abstract}

\section{Keywords : Misconceptions, Taylor series, nonelementary functions}

\section{INTRODUCTION}

The integral which cannot be expressed using elementary functions in finite form are called nonelementary integrals (Kumar, 2012). The researches related to the solution of nonelementary integral have been conducted by several researchers (Conrad, 2005; Kasper, 1980; Marchisotto \& Zakery, 1994; McMahon, 2015; Rosenlicht, 1972; Williams, 1993; Yadav, 2015). According to the researches, the solution could be solved or unsolved dependent on the integral forms. Yadav and Sen $(2008,2013)$ have introduced six standard forms of indefinite nonintegrable functions (classically known as nonelementary functions).

The Taylor series approach in solving indefinite integral is not included in the techniques of integration in standard calculus text books and a precise description of Liouville's proof is beyond the 
scope of a first year calculus course. According to this problem, researcher found that there are several calculus text books couldnot to recognize the forms of nonelementary integral. It makes for several lecturers confused to obtain the solution by using the Taylor series to answer the questions from their students.

The lack of information related to the application of the Taylor Series in learing of integration techniques has led to the misconceptions. According to experiences of researcher, the missconceptions could be seen through this examples, i.e. $\int e^{x} \ln x d x, \int \frac{\ln (1+x)}{x} d x$, $\int \frac{\sin x}{x-\pi} d x$, and $\int \cot (3+\ln x) d x$. The solutions of the integrals was conducted by several lecturers are as follows respectively.

$$
\begin{aligned}
& \text { 1. } \int e^{x} \ln x d x=\int \ln x d e^{x} \\
& =e^{x} \ln x-\int \frac{e^{x}}{x} d x \\
& =e^{x} \ln x-\int\left[\frac{1+x+\left(\frac{x^{2}}{2 !}\right)+\left(\frac{x^{3}}{3 !}\right)+\ldots}{x}\right] d x \\
& \int e^{x} \ln x d x=e^{x} \ln x-\left[\ln x+x+\frac{1}{4} x^{2}+\frac{1}{18} x^{3}+C+\ldots\right] \\
& \text { 2. } \int \frac{\ln (1+x)}{x} d x=\int\left(\frac{x-\frac{x^{2}}{2}+\frac{x^{3}}{3}-\frac{x^{4}}{4}+\ldots}{x}\right) d x \\
& =\int\left(1-\frac{x}{2}+\frac{x^{2}}{3}-\frac{x^{3}}{4}+\ldots\right) d x \\
& \int \frac{\ln (1+x)}{x} d x=x-\frac{x^{2}}{4}+\frac{x^{3}}{9}-\frac{x^{4}}{16}+C-\ldots \\
& \text { 3. } \int \frac{\sin x}{x-\pi} d x=\int\left[\frac{x-\left(\frac{x^{3}}{3 !}\right)+\left(\frac{x^{5}}{5 !}\right)-\left(\frac{x^{7}}{7 !}\right)+\ldots}{x-\pi}\right] d x
\end{aligned}
$$




$$
\begin{aligned}
& =\int \frac{x}{x-\pi} d x-\frac{1}{3 !} \int \frac{x^{3}}{x-\pi} d x+\frac{1}{5 !} \int \frac{x^{5}}{x-\pi} d x-\ldots \\
= & \int\left(1+\frac{\pi}{x-\pi}\right) d x-\frac{1}{3 !} \int\left(x^{2}+\pi x+\pi^{2}+\frac{\pi^{3}}{x-\pi}\right) d x+\frac{1}{5 !} \int\left(\frac{x^{5}}{x-\pi}\right) d x-\ldots \\
= & x+\pi \ln (x-\pi)-\frac{1}{3 !}\left[\frac{x^{3}}{3}+\frac{\pi x^{2}}{2}+\pi^{2} x+\pi^{3} \ln (x-\pi)\right]+\frac{1}{5 !} \int\left(\frac{x^{5}}{x-\pi}\right) d x-\ldots
\end{aligned}
$$

The solution couldnot be solved by several lecturers. Meanwhile, for another one had a solution for the same problem, Let $u=x-\pi$, so $d u=d x$.

$$
\begin{aligned}
\int \frac{\sin x}{x-\pi} d x & =\int \frac{\sin (u+\pi)}{u} d u \\
& =\int\left[\frac{-u+\left(\frac{u^{3}}{3 !}\right)-\left(\frac{u^{5}}{5 !}\right)+\ldots}{u}\right] d u \\
& =\int\left[-1+\left(\frac{u^{2}}{3 !}\right)-\left(\frac{u^{4}}{5 !}\right)+\ldots\right] d u \\
& =-u+\frac{u^{3}}{3.3 !}-\frac{u^{5}}{5.5 !}+C-\ldots \\
\int \frac{\sin x}{x-\pi} d x & =-(x-\pi)+\frac{(x-\pi)^{3}}{3.3 !}-\frac{(x-\pi)^{5}}{5.5 !}+C-\ldots
\end{aligned}
$$

4. $\int \cot (3+\ln x) d x$, let $u=3+\ln x$, so $d x=e^{u-3} d u$

$$
\begin{aligned}
\int \cot (3+\ln x) d x & =\int \cot u e^{u-3} d u \\
& =e^{-3} \int\left[\frac{\cos u}{\sin u}\right] e^{u} d u \\
& =e^{-3} \int\left[\frac{1-\left(\frac{u^{2}}{2 !}\right)+\left(\frac{u^{4}}{4 !}\right)-\ldots}{u-\left(\frac{u^{3}}{3 !}\right)+\left(\frac{u^{5}}{5 !}\right)-\ldots}\right]\left[\frac{1}{1-u+\left(\frac{u^{2}}{2 !}\right)-\left(\frac{u^{3}}{3 !}\right)+\ldots}\right] d u
\end{aligned}
$$




$$
\begin{aligned}
& =e^{-3} \int\left[\frac{1}{u}+1+\frac{u}{3}+\ldots\right] d u \\
& =e^{-3}\left[\ln u+u+\left(\frac{u^{2}}{6}\right)+C+\ldots\right] \\
\int \cot (3+\ln x) d x & =e^{-3}\left[\ln (3+\ln x)+(3+\ln x)+\frac{(3+\ln x)^{2}}{6}+C+\ldots\right] .
\end{aligned}
$$

All the solution above was conducted by using the Taylor series approach. In some cases it is not possible to rely on the Taylor series. If the function is not infinitely differentiable. Even if a Taylor series can be generated, there is good possibility that it will diverge and not represent the function one is attemping to antidifferentiate (“http://en.m.wikipedia.org/wiki/Nonelementary_integral," 2016).

A preliminary study of the solutions could be started by learning the Taylor series therom. Let $f$ be a function with derivatives of all orders in some interval $(a-r, a+\eta)$. the Taylor series

$$
f(x)=f(a)+\frac{f^{\prime}(a)(x-a)}{1 !}+\frac{f^{\prime \prime}(a)(x-a)^{2}}{2 !}+\frac{f^{\prime \prime \prime}(a)(x-a)^{3}}{3 !}+\ldots
$$

represents the function $f$ on the interval $(a-r, a+r)$ if and only if

$$
\lim _{n \rightarrow \infty} R_{n}(x)=0 \text {, }
$$

where $R_{n}(x)$ is the remainder in the Taylor's formula

$$
R_{n}(x)=\frac{f^{n+1}(c)}{(n+1) !}(x-a)^{n+1}
$$

And $\mathrm{c}$ is some point in $(a-r, a+r)$ (Varberg, Purcell, \& Rigdon, 2011).

For the special case $a=0$, the Taylor series

$$
f(x)=\sum_{n=0}^{\infty} \frac{f^{(n)}(0)}{n !} x^{n}=f(0)+\frac{f^{\prime}(0)}{1 !}+\frac{f^{\prime \prime}(0)}{2 !}+\ldots
$$

This case arises frequently enough that is given the special name Maclaurin series (Stewart, 2009).

The Taylor series of $f$ function represents $f$ function itself for $\lim _{n \rightarrow \infty} R_{n}(x)=0$, hence $\int f(x) d x=\int\left[f(a)+\frac{f^{\prime}(a)(x-a)}{1 !}+\frac{f^{\prime \prime}(a)(x-a)^{2}}{2 !}+\ldots\right] d x$, so that $\int f(x) d x$ can 
be solved by using the Taylor series, and vice versa. Probably, all the solution of several lecturers still containing the remainder term, therefore, the solutions needs to be investigated.

\section{METHOD}

The critical analysis is used to reveal the misconceptions of integral solution based on the examples that have been explained in background of study previously, i.e. $\int e^{x} \ln x d x, \int \frac{\ln (1+x)}{x} d x, \int \frac{\sin x}{x-\pi} d x$, and $\int \cot (3+\ln x) d x$. Operationally, the steps are as follows:

1. Collecting and learning concepts related to the Taylor series and nonelementary functions.

2. Analysing of the integrand form and/or the part of integrand such that the Taylor series can be applied and meet the requirements to integrable.

3. Integrating the Taylor series as the final solution of indefinite integral for nonelementary functions.

\section{RESULTS AND DISCUSSIONS}

The critical analysis for the solution of $\int e^{x} \ln x d x$ related to the attention of remainder term in the Maclaurin series of $e^{x}$. A reason why several lecturers does not consider the remainder term is the Maclaurin series of $e^{x}$ convergent for all $x$. In education world, in terms of solving mathematics problems, all the requirement must be written clearly, therefore, the reason above is wrong, even if the final solution is correct. As for the correct solution, must be showed that $\lim _{n \rightarrow 0} R_{n}(x)=0$ or $\lim _{n \rightarrow 0} R_{n}(x) \neq 0$ as follows:

$$
\begin{aligned}
& g(x)=e^{x} \quad g(0)=1 \\
& g^{\prime}(x)=e^{x} \quad g^{\prime}(0)=1 \\
& g^{n+1}(x)=e^{x} \quad g^{n+1}(0)=1 \\
& 1+x+\frac{x^{2}}{2 !}+\frac{x^{3}}{3 !}+\ldots+R_{n}(x) . \\
& R_{n}(x)=\frac{g^{(n+1)}(c)}{(n+1) !} x^{n+1}
\end{aligned}
$$




$$
\begin{aligned}
& \lim _{n \rightarrow \infty}\left|R_{n}(x)\right| \leq \lim _{n \rightarrow \infty}\left|\frac{1}{(n+1) n !} x^{n} \cdot x\right| \\
& \lim _{n \rightarrow \infty} \frac{1}{n+1}\left|\frac{x^{n}}{n !}\right||x|,
\end{aligned}
$$

where $\frac{x^{n}}{n !}$ is the $n$th term of a convergent series $\sum \frac{x^{n}}{n !}$, so that $\lim _{n \rightarrow \infty} \frac{x^{n}}{n !}=0$. Then $\lim _{n \rightarrow \infty}\left|R_{n}(x)\right| \leq 0.0 \cdot|x|=0$, since $|x|$ exist. So $\lim _{n \rightarrow \infty} R_{n}(x)=0$. Hence, the Maclaurin series of $e^{x}$ integrable and $\int e^{x} \ln x d x$ is solved as follows:

$\int e^{x} \ln x d x=e^{x} \ln x-\int\left(\frac{1}{x}+1+\frac{x}{2 !}+\frac{x^{2}}{3 !}+\ldots\right) d x=e^{x} \ln x-\left(\ln x+x+\frac{x^{2}}{4}+\frac{x^{3}}{18}+C+\ldots\right)$.

The solution of $\int \frac{\ln (1+x)}{x} d x$ also has a similar problem. The selection of the Maclaurin series to solve the integral was properly. But, there exist a misconception too, that is, pay no attention of rhe remainder term. If the solution is pay attention to the remainder term, then, the series will not represent of $\ln (1+x)$, so that, the integral unsolved using the Taylor series. The proof are as follow:

$$
\begin{aligned}
& \ln (1+x)=x-\frac{x^{2}}{2}+\frac{x^{3}}{3}-\ldots+\frac{(-1)^{n+1}}{n} x^{n}+R_{n}(x) \\
& f^{(n+1)}(c)=(-1)^{n+2}=(-1)^{n} \\
& \left|R_{n}(x)\right| \leq\left|\frac{(-1)^{n}}{n+1} x^{n+1}\right| \\
& \lim _{n \rightarrow \infty}\left|R_{n}(x)\right| \leq \lim _{n \rightarrow \infty}\left|\frac{(-1)^{n}}{n+1} x^{n+1}\right|,
\end{aligned}
$$

$\frac{(-1)^{n}}{n+1} x^{n+1}$ is the $n$th term of a series $\sum \frac{(-1)^{n}}{n+1} x^{n+1}$. Absolute ratio test can be used to prove that the series divergent, i.e. 
$p=\lim _{n \rightarrow \infty}\left|\frac{(-1)^{n+1}}{n+2} x^{n+2} \frac{n+1}{(-1)^{n} x^{n+1}}\right|$
$p=\lim _{n \rightarrow \infty}\left|\frac{(-1)^{n}(-1) x^{n+1} x}{n+2} \cdot \frac{n+1}{(-1)^{n} x^{n+1}}\right|=\lim _{n \rightarrow \infty} \frac{n+1}{n+2}|x|=|x|$ $\lim _{n \rightarrow \infty}\left(\frac{(-1)^{n} x^{n+1}}{n+1}\right)=|x|$, then, $\lim _{n \rightarrow \infty} R_{n}(x)=|x| \neq 0$. The series is convergent of an interval $|x|<1$ or $-1<x<1$, and divergent of an interval $|x|>1$, therefore, $\int \frac{\ln (1+x)}{x} d x$ unsolved using the Taylor series.

The critical analysis for the first solution of $\int \frac{\sin x}{x-\pi} d x$ revealed about the inability to choose the Taylor series that must be used, so that, the integral unsolved due to the Taylor series about $x=$ 0 still containing the integral form. As for the second solution, the critical analysis revealed a misconception related to the technique of supposition to determine the Taylor series of $f$ function. The mistake related to the derivative of $f$ function. Obviously, the derivative of a function before supposition is not equal to the derivative after supposition, therefore, the Maclaurin series expansion of $f(x)$ based on the Taylor series also a misconception, since the supposition of $u=x-\pi$, where $u \neq 0$. Another misconception for second solution is a pay no attention to the remainder term of the Taylor series. If the remainder term is $R_{n}(x)=0$, then, must be showed that $\lim _{n \rightarrow \infty} R_{n}(x)=0$ or $\lim _{n \rightarrow \infty} R_{n}(x) \neq 0$, therefore, must be known the Taylor series about $x=\pi$ of the function and the form of $R_{n}(x)$. The Taylor series expansion for $\sin x$ about $x=\pi$ are as follows:

$$
\begin{array}{ll}
g(x)=\sin x & g(\pi)=\sin \pi=0 \\
g^{\prime}(x)=\cos x & g^{\prime}(\pi)=\cos \pi=-1 \\
g^{\prime \prime}(x)=-\sin x & g^{\prime \prime}(\pi)=-\sin \pi=0 \\
g^{\prime \prime \prime}(x)=-\cos x & g^{\prime \prime \prime}(\pi)=-\cos \pi=1
\end{array}
$$




$$
\begin{aligned}
& -(x-\pi)+\frac{(x-\pi)^{3}}{3 !}-\frac{(x-\pi)^{6}}{5 !}+\ldots+R_{n}(x) \\
& R_{n}(x)=\frac{g^{(n+1)}(x)}{(n+1) !}(x-\pi)^{n+1} \\
& \left|g^{(n+1)}(x)\right|=|\cos x| \text { or }\left|g^{(n+1)}(x)\right|=|\sin x|,\left|g^{(n+1)}(c)\right|=1 \\
& \lim _{n \rightarrow \infty}\left|R_{n}(x)\right| \leq \lim _{n \rightarrow \infty}\left|\frac{(x-\pi)^{n+1}}{(n+1) !}\right| \\
& \lim _{n \rightarrow \infty}\left|\frac{(x-\pi)^{n}(x-\pi)}{(n+1) n !}\right| \leq \lim _{n \rightarrow \infty} \frac{1}{n+1}\left|\frac{(x-\pi)^{n}}{n !}\right| x-\pi \mid,
\end{aligned}
$$

where $\frac{(x-\pi)^{n}}{n !}$ is the $n$th term of a convergent series $\sum \frac{(x-\pi)^{n}}{n !}$, so that $\lim _{n \rightarrow \infty} \frac{(x-\pi)^{n}}{n !}=0$. Absolute ratio test can be used to prove that the series convergent, i.e.

$$
\begin{aligned}
& p=\lim _{n \rightarrow \infty}\left|\frac{(x-\pi)^{n+1}}{(n+1) n !} \cdot \frac{n !}{(x-\pi)^{n}}\right| \\
& p=\lim _{n \rightarrow \infty}\left|\frac{(x-\pi)^{n}(x-\pi)}{(n+1) n !} \cdot \frac{n !}{(x-\pi)^{n}}\right| \\
& p=\frac{1}{n+1}|x-\pi|=0|x-\pi|=0<1 \quad, \quad \text { since }|x-\pi| \quad \text { exist, }
\end{aligned}
$$
so $\lim _{n \rightarrow \infty}\left|R_{n}(x)\right| \leq 0.0 .|x-\pi|=0$, then $\lim _{n \rightarrow \infty} R_{n}(x)=0$, therefore, the Taylor series expansion about $x=\pi$ integrable and $\int \frac{\sin x}{x-\pi} d x$ can be solved as follows:

$$
\int \frac{\sin x}{x-\pi} d x=\int\left[-1+\frac{(x-\pi)^{2}}{3 !}-\frac{(x-\pi)^{4}}{5 !}+\ldots\right] d x=-x+\frac{(x-\pi)^{3}}{3.3 !}-\frac{(x-\pi)^{5}}{5.5 !}+C-\ldots
$$


The critical analysis for the solution of $\int \cot (3+\ln x) d x$ are as follows:

1. Changing the integrand form through supposition and the Maclaurin series expansion of $\cos u, \sin u$, and $e^{-u}$ are the same mistake to the second solution of $\int \frac{\sin x}{x-\pi} d x$ previously.

2. Assumption that the Taylor series expansion of $\cot u$ equal to the Taylor series expansion of $\cos u$ divided by the Taylor series expansion of $\sin u$ is a misconception. The Maclaurin series expansion of cot $u$ does not exist. While the Maclaurin series expansion of $\cos u$ divided by the Maclaurin series expansion of $\sin u$ exist. The proof of this statement are as follows:

$$
\begin{array}{ll}
f(u)=\cot u & f(0)=\infty \\
f^{\prime}(u)=-\frac{1}{\sin ^{2} u} & f^{\prime}(0)=\infty \\
f^{\prime \prime}(u)=\cot u & f^{\prime \prime}(0)=\infty
\end{array}
$$

The Maclaurin series: $f(0)+f^{\prime}(0) u+f^{\prime \prime}(0) \frac{u^{2}}{2 !}+\ldots=\infty+\infty+\ldots$ (does not exist). As for the proof of the Maclaurin series expansion of $\cos u$ and $\sin u$ are as follows respectively:

$$
\begin{array}{ll}
g(u)=\cos u & g(0)=1 \\
g^{\prime}(u)=-\sin u & g^{\prime}(0)=0 \\
g^{\prime \prime}(u)=-\cos u & g^{\prime \prime}(0)=-1 \\
g^{\prime \prime \prime}(u)=\sin u & g^{\prime \prime \prime}(0)=0
\end{array}
$$

The Maclaurin series: $g(0)+g^{\prime}(0) u+g^{\prime \prime}(0) \frac{u^{2}}{2 !}+\ldots=1-\frac{u^{2}}{2 !}+\frac{u^{4}}{4 !}-\ldots \neq 0$ (exist).

$$
\begin{array}{ll}
h(u)=\sin u & h(0)=0 \\
h^{\prime}(u)=\cos u & h^{\prime}(0)=1 \\
h^{\prime \prime}(u)=-\sin u & h^{\prime \prime}(0)=0
\end{array}
$$




$$
h^{\prime \prime \prime}(u)=-\cos u \quad h^{\prime \prime \prime}(0)=-1
$$

The Maclaurin series: $h(0)+h^{\prime}(0) u+h^{\prime \prime}(0) \frac{u^{2}}{2 !}+\ldots=u-\frac{u^{3}}{3 !}+\frac{u^{5}}{5 !}-\ldots \quad \neq 0$ (exist), so that, the Maclaurin series expansion of $\cos u$ divided by the Maclaurin series expansion of $\sin u$ also exist.

3. Pay no attention to the remainder term. The form of $\cot (3+\ln x)$ could be expanded based on the Taylor series expansion, except about $x=0$ and $x=e^{-3}$. The Taylor series expansion about $x=a$ are as follows:

$$
\begin{array}{ll}
f(x)=\cot (3+\ln x) & f(a)=\cot (3+\ln a) \\
f^{\prime}(x)=\frac{-1}{x \sin ^{2}(3+\ln x)} & f(a)=\frac{-1}{a \sin ^{2}(3+\ln a)} \\
f^{\prime \prime}(x)=\frac{2 \cos (3+\ln x)+\sin (3+\ln x)}{x^{2} \sin ^{3}(3+\ln x)} & f^{\prime \prime}(a)=\frac{2 \cos (3+\ln a)+\sin (3+\ln a)}{a^{2} \sin ^{3}(3+\ln a)}
\end{array}
$$$$
\cot (3+\ln a)-\frac{(x-a)}{a \sin ^{2}(3+\ln a)}+\frac{2 \cos (3+\ln a)+\sin (3+\ln a)}{2 ! a^{2} \sin ^{3}(3+\ln a)}(x-a)^{2}-\ldots+R_{n}(x)
$$$$
R_{n}(x)=\frac{f^{(n+1)}(c)}{(n+1) !}(x-a)^{n+1} .
$$

The form of $f^{(n+1)}(c)$ very difficult to be determined and $\lim _{n \rightarrow \infty} R_{n}(x)=0$ or $\lim _{n \rightarrow \infty} R_{n}(x) \neq 0$ very difficult to prove, therefore, to obtain solution of $\int \cot (3+\ln x) d x$ is very difficult too.

\section{CONCLUSION}

The results revealed that there are several misconceptions in solving indefinite integral for nonelementary functions in this research, i.e. 
1. Pay no attention to the remainder term of the Taylor series so that integration of the Taylor series of $f$ function does not represent of $f$ function itself, where $f$ divergent of an interval $\left(\lim _{n \rightarrow \infty} R_{n}(x) \neq 0\right)$.

2. The selection of the Taylor series about $x=0$ or $x \neq 0$ is not suitable to the integrand form or the part of integrand, so that, the integral very difficult to be solved or even unsolved.

3. The way to obtain the Taylor series about $x=a$ by supposition technique.

4. Assumption that the Taylor series expansion of $f(x)$ equal to the Taylor series expansion of $g(x)$ divided by the Taylor series expansion of $h(x)$ could be applied for all condition.

\section{REFERENCES}

[1]. Conrad, B. (2005). Impossibility Theorems for Elementary Integration. Clay Mathematics Institute: Academy Colloquium Series. Retrieved from http://math.stanford.edu/ conrad/papers/finalint.pdf.

[2]. Kasper, T. (1980). Integration in Finite Terms: The Liouville Theory, Mathematics Magazine, 53(4), pp.195-201.

[3]. Kumar. V. (2012). Integral Calculus (2th ed.). Tata McGraw Hill Education Private Limited 7 West Patel Nagar, New Delhi 110008.

[4]. Marchisotto, E. A., \& Zakeri, G. (1994). An Invitation to Integration in Finite Terms, The College Mathematics Journal, 25(4), pp. 295-308.

[5]. McMahon, C. S. (2015). Impossible Integrals (Combinational approximating integral hybrids approximating non-elementary integrals with elementary integrals). The General Science Journal Retrieved February 7, 2016, from

[6]. Nonelementary Integral (n.d.) Retrieved February 9, 2016, from Wikipedia website, http://en.m.wikipedia.org/wiki/Nonelementary_integral.

[7]. Rosenlicht, M. (1972). Integration in Finite Terms, The American Mathematical Monthly, 79(9), pp. 963-972.

[8]. Stewart, J. (2009). Calculus Concepts \& Context (4th ed.). Cengange Learning. Inc. USA. Belmont, CA 94002-3098. 
[9]. Varberg, D., Edwin J. P., \& Steven E. R. (2006). Calculus (9th ed.). Prentice Hall.

[10]. Williams, D. P. (1993). Nonelementary Antiderivative. Department of Mathematics, Bradley Hall. Darmouth College, Hanover, NH. 03755-3551. USA.

[11]. Yadav, D. K. \& Sen, D. K. (2008). Revised paper on Indefinite Nonintegrable Functions, Acta Ciencia Indica, 34(3), pp.1383-1384.

[12]. Yadav, D. K. \& Sen, D. K. (2013). Proof of Second Standard Form of Nonelementary Functions. International Journal of Advanced Research in Computer Science and Software Engineering, 3(3), 2277 128X

[13]. Yadav, D. K. \& Sen, D. K. (2013). Proof of Fourth Standard Form of Nonelementary Functions. International Journal of Advanced Research in Computer Science and Software Engineering. 4(4), 2277 128X

[14]. Yadav, D. K. (2015). Early Basic Foundations of Modern Integral Calculus. International Journal of Education and Science Research Review. 2(2), 2348-6457. 